\title{
SOP Corona-Risikomanagement in der Zahnarztpraxis
}

\author{
Michaela Wolf
}

\author{
ABKÜRZUNGEN \\ AEMP Aufbereitungseinheit für Medizinprodukte \\ BZÄK Bundeszahnärztekammer \\ DAHZ Deutscher Arbeitskreis für Hygiene in der \\ Zahnmedizin \\ DGSV Deutsche Gesellschaft für Sterilgutversorgung \\ IDZ Institut der Deutschen Zahnärzte \\ LAGA Bund/Länder-Arbeitsgemeinschaft Abfall \\ LZÄK Landeszahnärztekammer \\ PSA persönliche Schutzausrüstung \\ RKI Robert-Koch-Institut \\ SOP Standardvorgehensweisen (engl. Standard \\ Operating Procedure) \\ TRBA Technische Regel für biologische Arbeitsstoffe \\ ZA behandelnder Zahnarzt \\ ZFA Zahnmedizinische Fachangestellte
}

\section{Einleitung}

Die Entscheidung über die Notwendigkeit einer Behandlung während der Corona-Pandemie trifft die Zahnärztin oder der Zahnarzt gemeinsam mit jedem Patienten. Grundsätzlich können Eingriffe notwendig werden, die nicht als Notfallbehandlung einzustufen sind. Zum Beispiel Eingriffe, welche die Beschwerden des Patienten kurz-, mittel- oder langfristig lindern oder eine Verschlimmerung der bestehenden Erkrankung verhindern.

Behandlungen von vulnerablen Patienten sind unter Beachtung der Hygiene- und Schutzmaßnahmen auf Akutund Notfallbehandlungen zu beschränken. Die Behandlung von Patienten mit Atemwegsinfektionen ist bis zum Abklingen des Infektes zu verschieben. Ist die Behandlung nicht verschiebbar, sind diese Patienten zum Hausarzt zum Ausschluss einer COVID-19-Erkrankung zu verweisen [1].

Die Notfallversorgung von COVID-19-Erkrankten, mit dringendem Verdacht oder unter Quarantäne stehenden Patienten ist vorzugsweise in den benannten Kliniken oder Schwerpunktpraxen vorzunehmen [2]. Die Einstufung der Patienten und ob eine Behandlung notwendig ist, erfolgt wenn möglich - bereits bei der telefonischen Terminvereinbarung der Patienten. Unterstützung bieten hierbei ein Screening-Fragebogen und die vom Institut der Deut- schen Zahnärzte (IDZ) erstellten Flussdiagramme. Der Screening-Fragebogen klärt, ob eine Infektion oder ein Verdacht vorliegt, beurteilt gleichzeitig die Dringlichkeit der Behandlung und gibt eine Anleitung für die Verfahrensweisen in Standardvorgehensweisen (SOP) [3].

\section{Praxismanagement}

\section{Allgemein}

Laut Robert Koch-Institut (RKI) besteht ein erhöhtes Risiko für eine Übertragung von SARS-CoV-2 auf Personal bei Aerosol produzierenden Vorgängen [4]. Die Entstehung und Verbreitung von Aerosolen sollen vermieden werden.

\section{PRAXIS}

Folgende Maßnahmen sollen angewendet werden [5]:

1. Die Verwendung von Ultraschallhandstücken, piezoelektrisch betriebenen Ultraschall- und Chirurgiegeräten vermeiden.

2. Die Verwendung von Pulverstrahlgeräten (z. B. „Air-Flow“) vermeiden.

3. Die Verwendung von Turbinen vermeiden.

4. Antiseptische Mundspülungen können dazu beitragen, eine Infektionsübertragung zu minimieren.

5. In Abhängigkeit von Art und Umfang der Exposition und des Infektionsrisikos entsprechende persönliche Schutzausrüstung konsequent und ordnungsgemäß tragen. Die zusätzliche Verwendung von Visieren/Schutzschilden bei der zahnärztlichen Behandlung kann die Sicherheit weiter erhöhen.

6. Weiterhin sollte jede Form der Behandlung von Risikogruppen (Senioren, multimorbide Patienten, immunsupprimierte oder immunreduzierte Patienten oder anders einschlägig gesundheitlich vorgeschädigte Patienten) auf ein absolut notwendiges Maß reduziert werden, besonders um Kontakte im Wartezimmer oder in der Praxis zu vermeiden.

Weitere Maßnahmen [6]:

7. Kofferdam als wirksame Barriere gegen die im Mund- und Rachenraum befindlichen Mikroorganismen.

8. Schutzmittel wie Handschuhe und ggf. Schutzkittel über die gesamte Behandlungszeit tragen, auf ordnungsgemäßen Sitz achten und Einhaltung der Griffdisziplin zur Gewährleistung der Barrierefunktion.

9. Aerosolvermeidung durch eine effiziente, hochvolumige Absaugung und 4-händiges Arbeiten. 


\section{Personal und Patienten}

Folgendes ist zu beachten [6]:

PERSONAL

- Auf jede körperliche Begrüßung verzichten.

- In den Behandlungsräumen Mindestabstand $>1,5 \mathrm{~m}$ zwischen den Mitarbeiter/innen einhalten.

- Alle Mitarbeiter sollten in der ZA-Praxis einen Mund-Nasen-Schutz tragen, auch im Gespräch miteinander. Rezeptionsbereiche können durch eine flüssigkeitsdichte Abtrennung geschützt werden.

- Regelmäßige Team-Besprechungen zur Erörterung, Festlegung und Anpassung der nötigen Maßnahmen.

\section{PATIENTEN}

- Informieren Sie die Patienten auf Ihrer Homepage und an der Eingangstür durch einen Aushang zum Thema „Corona“. Hinweisschilder gibt es als Download auf der Internetseite der BZÄK [7] und der LZÄK Hessen [8].

- Anzahl der wartenden Personen im Wartezimmer beschränken, Stuhlabstand mind. $2 \mathrm{~m}$.

- Begleitpersonen erwachsener Patienten bitten, die Praxis zu verlassen und die Patienten nach Behandlungsende vor der Praxis abzuholen.

- Patienten anhalten, sich die Hände nach dem Betreten und vor dem Verlassen der Praxis zu desinfizieren und möglichst wenige Oberflächen zu berühren. Dies gilt auch für Türklinken; wenn möglich mit dem Ellenbogen öffnen oder Türen soweit möglich offen stehen lassen.

- Zeitschriften und Spielzeug aus dem Wartebereich entfernen.

- Regelmäßig alle von Patienten berührten Oberflächen in Praxis und Wartezimmer desinfizieren; Behandlungsstuhl nach jedem Patienten.

\section{Behandlung von asymptomatischen Patienten, für die kein Verdacht besteht, mit SARS-CoV-2 infiziert zu sein}

Folgendes ist zu beachten [9]:

- Aerosol bildende Maßnahmen vermeiden (siehe [4])

- Schutzkleidung für ZA und ZFA:

- (normale) Arbeitskleidung

- Schutzbrille mit Seitenschutz

- Mund-Nasen-Schutz

- hygienische Händedesinfektion + unsterile Einmalhandschuhe

- Zusätzliche Maßnahmen (Beispiele):

- vor Behandlung orale Antisepsis

- Multifunktionsspritze ohne Luft-WasserSpray verwenden (nur Luft oder nur Wasser)

- wenn möglich, Verwendung von Kofferdam

\footnotetext{
Cave
}

Im Rahmen der Zunahme der Pandemie ist die Versorgung eines asymptomatischen SARS-CoV-2Trägers nicht auszuschließen. Bei nicht verschiebbaren Behandlungen mit massiv Aerosol bildenden Maßnahmen ist daher Schutzkleidung in Abhängigkeit von Art und Umfang der Exposition und des Infektionsrisikos für die Mitarbeiter (Gesichtsschutz) Visier, erregerdichter Schutzkittel, FFP2- oder FFP3Maske) anzulegen. 


\section{Behandlung von Patienten mit Verdacht auf eine Infektion mit SARS-CoV-2 (auch Patient in Quarantäne oder Kontaktperson)}

- Diagnostik oder Behandlung in der Regel in zahnmedizinischem Behandlungszentrum/Schwerpunktpraxis für Corona-Patienten; Terminvereinbarung und Überweisung einleiten [10].

- Ist eine Behandlung in einer Schwerpunktpraxis nicht möglich, sind bereits bei Verdacht die entsprechenden Schutz- und Hygienemaßnahmen wie bei bestätigtem positiven SARS-CoV-2Befund durchzuführen.

- Äußert der Patient den Verdacht erst an der Rezeption, ist ein Kontakt (Abstandsregel) zu vermeiden und dem Patienten ein Mund-NasenSchutz auszuhändigen.

- der Patient soll keine weiteren Räume (Wartezimmer) betreten und sich vor dem Verlassen der Praxis die Hände desinfizieren

- Kontaktflächen sind desinfizierend zu reinigen

- Behandlung nur in Schwerpunktpraxis

\section{Umgang mit unaufschiebbaren Akut- und Notfallbehandlungen bei Patienten mit Nachweis oder begründetem Ver- dacht auf SARS-CoV-2 oder Erkrankung (keine Behandlung in Schwerpunkt- praxis möglich)}

Bei der Behandlung sind besondere Schutz- und Hygienemaßnahmen notwendig [11]:

- Räumliche oder organisatorische Trennung des an COVID-19 erkrankten Patienten von den Patienten der Normalsprechstunde.

- Persönliche Schutzausrüstung für das Personal

- Langärmliger, flüssigkeitsabweisender Schutzkittel mit Rückenschluss und Abschlussbündchen

- Kopfhaube und ggf. Füßlinge

- Schutzbrille mit Seitenschutz/Visier

- Atemschutzmaske (FFP2/FFP3)

- Einmalhandschuhe

- An- und Ablegen der Schutzkleidung z. B. gemäß des Ablaufplans der BZÄK [12] und unter Berücksichtigung der Hinweise zum sicheren An- und Ablegen von Atemschutzmasken, Schutzbrillen und Einmalhandschuhen [13]
- Behandlungs- bzw. Isolierzimmer vorbereiten [14]

- Raum (Außenseite der Tür) kennzeichnen mit Hinweisschild "Isolierzimmer“

- Entfernen von nicht benötigten Gegenständen

- Wenn nicht entfernbar und nicht (später) wischdesinfizierbar: Abdeckung von Gegenständen und Oberfläche mit flüssigkeitsdichter Folie, da die Aerosolwolke bis zu $3 \mathrm{~m}$ im Umfeld der Behandlungseinheit nachweisbar ist

- Vollständige Vorbereitung aller für die Behandlung benötigten Instrumente und Materialien inklusive Behälter für Instrumenten- und Abfallentsorgung

- Wegeführung des Patienten [15]

- PSA zum Abholen des Patienten anlegen

- Patienten vor Betreten der Praxis Hände desinfizieren und Mund-Nasen-Schutz anlegen lassen

- Patienten darauf hinweisen, dass er nichts anfassen soll (Türen öffnen) und direkt ins Isolierzimmer bringen; wenn doch Flächen berührt wurden, diese desinfizierend reinigen

- elektronische Gesundheitskarte der/-s Patientin/-en entgegennehmen und desinfizieren; elektronische Gesundheitskarte einlesen und zurückgeben

- Behandlung - zusätzliche Schutzmaßnahmen [16]

- Behandlung auf Behandler und Assistenz und ggf. Springer beschränken

- Abdeckung der Patientenkleidung mit flüssigkeitsdichtem Tuch/Folie

- Patient: Ausziehen des Mund-Nasen-Schutzes und orale Antisepsis z. B. $\mathrm{H}_{2} \mathrm{O}_{2}$ (1,5\%) mit $30 \mathrm{ml}$ für 30 Sekunden

- Erhöhung des Infektionsschutzes durch Verwendung von Kofferdam

- möglichst Aerosolbildung vermeiden; je nach Exposition FFP3-Maske verwenden

- Behandlung durchführen mit desinfizierten und/oder sterilen Instrumenten (bestimmungsgemäßer Gebrauch)

- Nach der Behandlung [17]

- Entfernen des Abdecktuchs von der Patientenkleidung und Entsorgung in Abfall

- Fenster öffnen, mindestens 30 Minuten lüften

- Patient: Mund-Nasen-Schutz anlegen lassen und aus der Praxis begleiten

- Vor Begleitung des Patienten ist die PSA der ZFA teilweise abzulegen und zu erneuern (Kopfhaube, Schutzbrille/ Visier, Schutzkittel und Handschuhe ablegen, nach Händedesinfektion neue Handschuhe anlegen) und Patienten hinausführen

- ZA und ZFA legen PSA kontaminationsfrei vollständig ab

- Fenster geöffnet lassen, mindestens weitere 30 Minuten vor desinfizierender Reinigung lüften

- ZFA legt neue PSA komplett an (Handschuhe mit langen Stulpen DIN EN 374) und führt die desinfizierende Reinigung des Isolierzimmers mit begrenzt viruzidem Flächendesinfektionsmittel durch

- Entsorgung des Abfalls

- Aufbereitung der Instrumente: Transport der Instrumente in geschlossenem Container; vor Transport Container von außen wischdesinfizieren 
- Abfallentsorgung [18, 19]

- Für nicht flüssige Abfälle (z. B. Schutzkleidung, Tupfer) bestehen keine besonderen Anforderungen für die Abfallsammlung. Solche Abfälle sind der Abfallschlüsselnummer 180104 der LAGA-Richtlinie (Entsorgung von Abfällen aus Einrichtungen des Gesundheitsdienstes) zugeordnet. Für die Entsorgung sind verschlossene und reißfeste Plastiksäcke ausreichend. Spitze und scharfe Gegenstände sind in durchstichsicheren Einwegbehältnissen zu sammeln.

- Für flüssige Abfälle, z. B. Absaugmaterial, sind aus infektionspräventiver Sicht besondere Anforderungen an Sammlung und Entsorgung notwendig. Solche Abfälle sind der Abfallschlüsselnummer 180103 der LAGA-Richtlinie zugeordnet. Hierfür sind spezielle Behältnisse bzw. bauartgeprüfte Gefahrgutverpackung mit Kennzeichnung durch „Biohazard“-Symbol gemäß TRBA 250 notwendig.

\section{Hinweis [20]}

Aktuell besteht immer noch eine Knappheit auf dem Weltmarkt an Schutzausrüstung, insbesondere MundNasen-Schutz und Atemschutzmasken. Das RKI veröffentlichte daher Mitte April das Papier „Mögliche Maßnahmen zum Ressourcen-schonenden Einsatz von MundNasen-Schutz (MNS) und FFP-Masken in Einrichtungen des Gesundheitswesens bei Lieferengpässen im Zusammenhang mit der neuartigen Coronavirus-Erkrankung COVID-19“. Dieses Papier ist vorerst gültig bis 31.08.2020 und ist nur in Notfallsituationen anzuwenden. Es erlaubt die Wiederverwendung von Einmalmaterial unter bestimmten Voraussetzungen. Vor dem Einführen der Wiederverwendung von MNS und FFP2- und FFP3-Masken sollte eine fachkundige Gefährdungsbeurteilung bzw. Risikobewertung durch den Arbeitgeber vor Ort unter Einbeziehung des Hygienefachpersonals, des betriebsärztlichen Dienstes und ggf. in Rücksprache mit dem zuständigen Gesundheitsamt erfolgen.
Folgende Punkte sind bei der Wiederverwendung zu beachten:

- patientenbezogene oder patientenübergreifende Wiederverwendung von MNS und FFP-Masken während einer Schicht/eines Arbeitstages nur durch dieselbe Person

- KEINE Wiederverwendung bei operativen Eingriffen

- sofortiger Wechsel des MNS bzw. der FFP-Masken bei (vermuteter) Kontamination bzw. Durchfeuchtung weiterhin notwendig

- KEINE Wiederverwendung bei Tätigkeiten an infektiösen Patienten mit ausgeprägter Exposition zu Aerosolen

Die Wiederverwendung von MNS und FFP-Maske erfordert einen sachgerechten Umgang. Bei unsachgemäßem Umgang besteht ein erhöhtes Infektionsrisiko für das Personal. Folgendes ist zu beachten:

- Beim Absetzen, Ablegen und Wiederaufsetzen der Maske/des MNS unbedingt darauf achten, dass hierdurch eine Kontamination der Maske/des MNS (vor allem der Innenseite) bzw. eine Kontamination des Gesichtes verhindert wird (siehe [13]).

- Aufbewahrung/Zwischenlagerung der Maske/des MNS zur Vermeidung der Kontamination der Innenseite sowie Verschleppung auf andere Oberflächen

- trocken an der Luft, nicht in geschlossenen Behältern

- an einem festgelegten, nicht für Publikumsverkehr zugänglichen Ort

- die gebrauchte Maske/den gebrauchten MNS eindeutig einer Person zuordnen durch Markieren der Masken am Halteband

- benutzte Einweg-FFP-Masken/MNS nicht mit Desinfektionsmittel reinigen oder desinfizieren, da dies die Funktionalität der Maske negativ beeinflusst

- beim erneuten Aufsetzen neue Handschuhe anlegen und die Handschuhe vor erneutem Patientenkontakt entsorgen

- Masken/MNS, deren Innenfläche durch Fehler bei der Handhabung möglicherweise kontaminiert wurden, sind zu entsorgen

- den Ort, an dem die Zwischenlagerung erfolgt, unmittelbar nach Entnahme der Maske/des MNS sachgerecht desinfizieren 


\section{Wiederaufbereitung von Atemschutzmasken (FFP) [21]}

Sind Masken nicht lieferbar, kann eine Wiederaufbereitung der FFP-Maske unter bestimmten Voraussetzungen erfolgen. Die Deutsche Gesellschaft für Sterilgutversorung (DGSV) hat am 01.04.2020 eine Stellungnahme zur Wiederaufbereitung veröffentlicht: „Wiederaufbereitung“ von Schutzmasken im Rahmen der „Coronapandemie“ mit Bezug auf die Veröffentlichung des Dokumentes „Vorlage für den Krisenstab der Bundesregierung/Einsatz von Schutzmasken in Einrichtungen des Gesundheitswesens".

Es wird ausdrücklich darauf hingewiesen, dass die Aufbereitung von Atemschutzmasken, die eigentlich ein Einmalprodukt sind, als „Ultima Ratio“ zu verstehen ist. Grundsätzlich ist das Ziel, genügend Schutzmasken zum Schutz der gefährdeten Mitarbeiter zur Verfügung zu stellen.

Die DGSV hält die Aufbereitung mit trockener Hitze nicht für sicher, hygienisch und praktikabel und verweist auf die Aufbereitung in einer Aufbereitungseinheit für Medizinprodukte (AEMP). Etablierte und valide Verfahren sind z. B. das Vakuum-Dampf-Vakuum-Verfahren zur Dampfdesinfektion (VDV-Verfahren bei $105^{\circ} \mathrm{C}$ ) oder die Dampfsterilisation (z.B. fraktioniertes Vakuumverfahren bei $\left.121^{\circ} \mathrm{C} / 20 \mathrm{~min}\right)$. Diese Programme sind jederzeit und kurzfristig durch die Hersteller der Sterilisatoren aktivierbar.

\section{Schlussbemerkung}

Die Entwicklungen in der Corona-Pandemie sind sehr schnelllebig: „dynamisches Geschehen“. Das bedeutet, dass sich das Risikomanagement täglich ändern kann. Gründe hierfür sind:

- aktuelle wissenschaftliche Erkenntnisse (Übertragungswege, Infektiosität, Infektionsraten ...)

- Mangel an Schutzkleidung

- besondere Situationen, die erst im Praxisalltag deutlich werden

- behördliche Anordnungen

\section{Merke}

Das Hygienemanagement bedarf daher eines ständigen Abgleichs mit den aktuellen Informationen des Robert Koch-Instituts (RKI), der Bundes- und Landeszahnärztekammer (BZÄK, LZÄK), dem Institut der Deutschen Zahnärzte (IDZ), der Ministerien und Behörden. Hierfür ist es sinnvoll, eine Person im Team, z. B. die Hygienebeauftragte, zu benennen.
Interessenkonflikt

Die Autorin gibt an, dass kein Interessenkonflikt besteht.

Autorinnen/Autoren

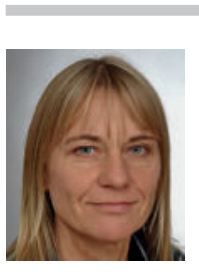

\section{Michaela Wolf}

Hygienefachkraft am Institut für Hygiene und Umweltmedizin, Universitätsklinikum Gießen und Marburg GmbH, Gießen.

Korrespondenzadresse

\section{Michaela Wolf}

Institut für Hygiene u. Umweltmedizin

Universitätsklinikum Gießen und Marburg GmbH

Friedrichstraße 16

35390 Gießen

Michaela.Wolf@uk-gm.de

\section{Literatur}

[1] Empfehlungen der Bundeszahnärztekammer; Corona-Risikomanagement; Behandlung von Patienten, für die kein dringender Verdacht besteht mit SARS-CoV-2 infiziert zu sein. Im Internet: https://www.bzaek.de/berufsausuebung/sars-cov2covid-19/risikomanagement.html; Stand: 15.04.2020

[2] DAHZ. Stellungnahme: SARS-COV-2/Covid-19 - Risikomanagement in Zahnarztpraxen. Im Internet: http://dahz.org/ wp-content/uploads/2020/04/DAHZ-Stellungnahme-Corona20.04.2020.pdf; Stand: 20.04.2020

[3] Institut der Deutschen Zahnärzte. System von Standardvorgehensweisen für Zahnarztpraxen während der CoronavirusPandemie. Im Internet: https://www.idz.institute/publikationen/sonstiges/system-von-standardvorgehensweisen-fuerzahnarztpraxen-waehrend-der-coronavirus-pandemie.html; Stand 24.04.2020

[4] Empfehlungen des Robert Koch-Instituts; SARS-CoV-2 Steckbrief zur Coronavirus-Krankheit-2019 (COVID-19); Kapitel 1: Übertragungswege. Im Internet: https://www.rki.de/DE/Content/InfAZ/N/Neuartiges_Coronavirus/Steckbrief.html\#doc 13776792bodyText1; Stand 07.05.2020

[5] Empfehlungen der Bundeszahnärztekammer und der Zahnärztekammern. Coronavirus: Merkblatt Corona-Risikomanagement ergänzt. Im Internet: https://www.lzkh.de/ueberuns/aktuelles; Stand: 23.03.2020

[6] Empfehlungen der Bundeszahnärztekammer; Corona-Risikomanagement; Behandlung von Patienten, für die kein dringender Verdacht besteht mit SARS-CoV-2 infiziert zu sein. Im Internet: https://www.bzaek.de/berufsausuebung/sars-cov2covid-19/risikomanagement.html; Stand: 09.04.2020

[7] Bundeszahnärztekammer. Praxisaushang Stop (März 2020). Im Internet: https://www.bzaek.de/fileadmin/PDFs/b/STOP-. pdf; Stand: 17.03 .2020

[8] Landeszahnärztekammer. Coronavirus Hinweis für die Praxistür. Im Internet: https://www.lzkh.de/ueber-uns/aktuelles; Stand: 01.04 .2020 
[9] Institut der Deutschen Zahnärzte. System von Standardvorgehensweisen für Zahnarztpraxen während der CoronavirusPandemie; Seite 29. Im Internet: https://www.idz.institute/ publikationen/sonstiges/system-von-standardvorgehensweisen-fuer-zahnarztpraxen-waehrend-der-coronavirus-pandemie.html; Stand 24.04.2020

[10] Institut der Deutschen Zahnärzte. System von Standardvorgehensweisen für Zahnarztpraxen während der CoronavirusPandemie; Seite 32. Im Internet: https://www.idz.institute/ publikationen/sonstiges/system-von-standardvorgehensweisen-fuer-zahnarztpraxen-waehrend-der-coronavirus-pandemie.html; Stand 24.04.2020

[11] Bundeszahnärztekammer. Risikomanagement. Im Internet: https://www.bzaek.de/berufsausuebung/sars-cov-2covid-19/ risikomanagement.html\#c10113; Stand 21.04.2020

[12] Bundeszahnärztekammer. Risikomanagement. Ablaufplan zum An- und Ablegen von Persönlicher Schutzausrüstung. Im Internet: https://www.bzaek.de/berufsausuebung/sars-cov2covid-19/risikomanagement.html\#c10113; Stand 21.04.2020

[13] Robert Koch-Institut. Hinweise zum beispielhaften An- und Ablegen von PSA für Fachpersonal. Im Internet: https://www. rki.de/DE/Content/InfAZ/N/Neuartiges_Coronavirus/ PSA_Fachpersonal/Dokumente_Tab.html; Stand: 16.04.2020

[14] Institut der Deutschen Zahnärzte. System von Standardvorgehensweisen für Zahnarztpraxen während der CoronavirusPandemie; Seite 45. Im Internet: https://www.idz.institute/ publikationen/sonstiges/system-von-standardvorgehensweisen-fuer-zahnarztpraxen-waehrend-der-coronavirus-pandemie.html; Stand 24.04.2020

[15] Institut der Deutschen Zahnärzte. System von Standardvorgehensweisen für Zahnarztpraxen während der CoronavirusPandemie; Seite 48. Im Internet: https://www.idz.institute/ publikationen/sonstiges/system-von-standardvorgehensweisen-fuer-zahnarztpraxen-waehrend-der-coronavirus-pandemie.html; Stand 24.04.2020

[16] Institut der Deutschen Zahnärzte. System von Standardvorgehensweisen für Zahnarztpraxen während der CoronavirusPandemie; Seite 50. Im Internet: https://www.idz.institute/ publikationen/sonstiges/system-von-standardvorgehensweisen-fuer-zahnarztpraxen-waehrend-der-coronavirus-pandemie.html; Stand 24.04.2020
[17] Institut der Deutschen Zahnärzte. System von Standardvorgehensweisen für Zahnarztpraxen während der CoronavirusPandemie; Seite 51-56. Im Internet: https://www.idz.institute/publikationen/sonstiges/system-von-standardvorgehensweisen-fuer-zahnarztpraxen-waehrend-der-coronavirus-pandemie.html; Stand 24.04.2020

[18] Robert Koch-Institut. Empfehlungen des RKI zu Hygienemaßnahmen im Rahmen der Behandlung und Pflege von Patienten mit einer Infektion durch SARS-CoV-2. Im Internet: https:// www.rki.de/DE/Content/InfAZ/N/Neuartiges_Coronavirus/ Hygiene.html; Stand 24.04.20

[19] Technische Regel für Biologische Arbeitsstoffe 250; Biologische Arbeitsstoffe im Gesundheitswesen und in der Wohlfahrtspflege; Anhang 8; Ausgabe März 2014. Im Internet: https://www.baua.de/DE/Angebote/Rechtstexte-und-Technische-Regeln/Regelwerk/TRBA/pdf/TRBA-250.pdf?_blob= publicationFile\&v=4; Stand: 30.04 .2020

[20] Robert Koch-Institut. Mögliche Maßnahmen zum Ressourcenschonenden Einsatz von Mund-Nasen-Schutz (MNS) und FFPMasken in Einrichtungen des Gesundheitswesens bei Lieferengpässen im Zusammenhang mit der neuartigen Coronavirus-Erkrankung COVID-19. Im Internet: https://www.rki.de/ DE/Content/InfAZ/N/Neuartiges_Coronavirus/Ressourcen_schonen_Masken.pdf?_blob=publicationFile; Stand: 14.04.2020

[21] Deutsche Gesellschaft für Sterilgutversorgung. Stellungnahme zur "Wiederaufbereitung“ von Schutzmasken im Rahmen der "Coronapandemie“ mit Bezug auf die Veröffentlichung des Dokumentes „Vorlage für den Krisenstab der Bundesregierung/Einsatz von Schutzmasken in Einrichtungen des Gesundheitswesens“. Im Internet: https://www.dgsv-ev.de/wp-content/uploads/2020/04/Stellungnahme-DGSV-Dekontamination-FFP-Masken-3.pdf; Stand: 05.04.2020

Bibliografie

DOI https://doi.org/10.1055/a-1166-8996

Zahnmedizin up2date 2020; 14: 185-190

(c) Georg Thieme Verlag KG Stuttgart · New York ISSN 1865-0457 